\title{
The excitation of rogue waves in a variable medium: an experimental study on the interaction of water waves and currents
}

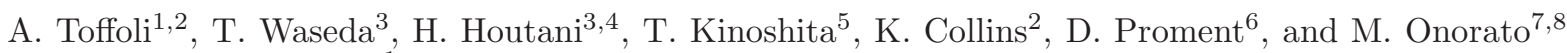 \\ ${ }^{1}$ Centre for Ocean Engineering Science and Technology, \\ Swinburne University of Technology, P.O. Box 218, Hawthorn, 3122 Vic., Australia; \\ ${ }^{2}$ School of Marine Science and Engineering, Plymouth University, Plymouth PL4 8AA, UK; \\ ${ }^{3}$ Graduate School of Frontier Sciences, University of Tokyo, Kashiwa, Chiba 27r-8563, Japan; \\ ${ }^{4}$ National Maritime Research Institute, Shinkawa, Mitaka-shi, Tokyo 180-0004, Japan; \\ ${ }^{5}$ Institute of Industrial Science, University of Tokyo, Tokyo, Japan; \\ ${ }^{6}$ School of Mathematics, University of East Anglia, Norwich, NR4 7TJ, UK; \\ ${ }^{7}$ Dipartimento di Fisica, Università degli Studi di Torino, Via Pietro Giuria 1, 10125 Torino, Italy \\ ${ }^{8}$ INFN, Sezione di Torino, Via Pietro Giuria 1, 10125 Torino, Italy.
}

(Dated: September 8, 2018)

\begin{abstract}
We show experimentally that a stable wave propagating into a region characterized by an opposite current may become modulationaly unstable. Experiments have been performed in two independent wave tank facilities; both of them are equipped with a wavemaker and a pump for generating a current propagating in the opposite direction with respect to the waves. The experimental results support a recent conjecture based on a current-modified Nonlinear Schrödinger equation which establishes that rogue waves can be triggered by non-homogeneous current characterized by a negative horizontal velocity gradient.
\end{abstract}

PACS numbers:

Ocean waves are characterized by a statistically small steepness and often (but not always, see for example [1]) a weakly nonlinear approach is sufficient to capture some of the intriguing aspects hidden in the fully nonlinear primitive equations. This weakly nonlinear approach is also shared by other fields of physics such as for example nonlinear optics [2] and plasma physics [3] where small parameters can be individuated and asymptotic expansions can be used to simplify the original equations. If the considered physical process is not only weakly nonlinear but also narrow-banded then the lion's share is played by the Nonlinear Schrödinger equation (NLS). Being an exactly integrable equation via the Inverse Scattering Transform [4], bizarre analytical solutions have been found in the past: besides traveling waves, breathers or multi-breather solutions have been found [5 7] and observed in hydrodynamic [8, 9], nonlinear optics 10, 11] and plasma [12] experiments. Starting from [13, 14], such solutions have been considered as prototypes of rogue waves. The early stages of the so called Akhmediev breather solution [6] describes the exponential growth of a slightly perturbed plane waves, i.e., it corresponds to the classical modulational instability process [15]. For water waves in infinite water depth, the instability is active when $\varepsilon N \geq 1 / \sqrt{2}$, where $\varepsilon=k_{0} a_{0}$ is the initial steepness of the plane wave, $k_{0}$ the wavenumber, $a_{0}$ its amplitude and $N=\omega_{0} / \Delta \Omega$ is the number of waves under the modulation; $\omega_{0}$ is the angular frequency corresponding to the carrier wavenumber $k_{0}$ and $\Delta \Omega$ the angular frequency of the modulation.

The whole picture is by now pretty well understood and relies on the fact that the medium in which waves propagate is homogeneous. In terms of the NLS equation this means that the coefficients of the dispersive and nonlinear terms do not depend on the spatial co- ordinates. Much more complicated and intriguing is the case in which the medium changes its properties along the direction of propagation of the waves. This situation is much more difficult to treat in terms of simplified models because it turns out that in general the resulting modified NLS does not share the property of integrability as the standard NLS and analytical breathers solutions can be found only in special cases (see some examples in 16 18]).

In the oceanographic context, the non-homogeneity of the medium is mostly due to currents or bottom topography. In this Letter we will consider, from an experimental point of view, the interaction of waves and currents and the consequent formation of rogue waves. Wave-current interaction and its effect on wave instability have been the subject of a number studies over the past decades 19 26]. Only recently, however, has the rogue wave formation process induced by an opposite current gradient been studied numerically on the basis of the modified NLS equation [27 30]. Specifically, 28] applied a transformation to the one dimensional current-modified NLS equation derived in 27] to obtain the following NLS-type of equation:

$$
\frac{\partial B}{\partial x}+i \frac{k_{0}}{\omega_{0}^{2}} \frac{\partial^{2} B}{\partial t^{2}}+i k_{0}^{3} \exp \left(-\Delta U / c_{g}\right)|B|^{2} B=0,
$$

where $c_{g}$ is the group velocity, $\Delta U=U(x)-U(0)$, with $U(x)$ the velocity of the current at position $x$ and $\mathrm{U}(0)$ is the current at $x=0$. For simplicity, we will consider the physical case of a wave generated in a region of zero current, $U(0)=0$, that enters into a region where an opposite current starts increasing its speed (in absolute value) and then adjusts to some constant value $U_{0}$. Note that in this case the coefficient of the nonlinear term of 
equation (1) increases as the waves enter into the current up to a certain value and then remains constant. The net effect is therefore an increase of the nonlinearity of the system. Numerical simulations of the current modified NLS equation presented in [28] showed that an envelope of an initially stable wave train becomes unstable after entering in the current region. As a result, the maximum amplitude shows a growing trend for increasing the ratio $U_{0} / c_{g}$, corroborating the idea that an originally stable plane wave is transformed into a breather by the presence of a current.

In [30] it was noted that the modified NLS equation proposed in [27] and used in [28] does not preserve wave action (see [21]). To the lowest order, conservation of wave action can be accounted for in equation (1) by simply multiplying the ratio $U_{0} / c_{g}$ by a factor 2 . Based on this modification, a prediction for the maximum wave amplitude obeys the following equation:

$$
\frac{A_{\max }}{\sqrt{E}}=1+2 \sqrt{1-\left[\frac{\exp \left(U_{0} / c_{g}\right)}{\sqrt{2} \varepsilon N}\right]^{2}},
$$

where $A_{\max }$ is the maximum wave amplitude achieved in the region of constant current and $\sqrt{E}$ is standard deviation of the wave envelope once the current has reached its maximum constant value. In [30] a derivation of a modified NLS equation based on an Hamiltonian formulation of surface gravity waves has been performed. A similar prediction as the one in (2) has been proposed and takes the following form:

$$
\frac{A_{\max }}{\sqrt{E}}=1+2 \sqrt{1-\left[\frac{\left(1+\sqrt{1+2 U_{0} / c_{g}}\right)^{4}}{\sqrt{2} \varepsilon N 16\left(1+2 U_{0} / c_{g}\right)^{1 / 4}}\right]^{2}} .
$$

Note that, to the lowest order, an expression equivalent to (3) can be derived from equation (1) by multiplying the ratio $U_{0} / c_{g}$ by a factor 3 .

In this Letter we present two independent sets of laboratory experiments that were conducted in the wave flume of Plymouth University and in the narrow directional wave basin at the Ocean Engineering Tank of the University of Tokyo. The wave flume at Plymouth University is $35 \mathrm{~m}$ long and $0.6 \mathrm{~m}$ wide with a uniform water depth of $0.75 \mathrm{~m}$. The facility is equipped with a piston wavemaker with active force absorption at one side and a passive absorber panel at the other end; only unidirectional propagation is allowed. The flume is also equipped with a pump for the generation of a background current up to $0.5 \mathrm{~m} / \mathrm{s}$, which can follow or oppose the wave direction of propagation (only an opposing current was used for the present study though). One of the inlet/outlet is located nearby the absorber, while the other is at a distance of about $2.5 \mathrm{~m}$ from the wavemaker. This particular configuration allows waves to be generated outside the current field and propagate for a few wavelengths before encountering a current gradient. The wave field was monitored with 10 wave probes equally spaced along the tank, while the velocity field was monitored with two
Acoustic Doppler Velocimeters (ADV) properly seeded. A survey of the current revealed a fairly uniform flow both in space and time. An example of longitudinal and vertical profiles of the average horizontal velocity is presented in Figs. 17a and 10.

The Ocean Engineering Tank of the Institute of Industrial Science, University of Tokyo (Kinoshita Laboratory and Rheem Laboratory), is $10 \mathrm{~m}$ wide, $50 \mathrm{~m}$ long and $5 \mathrm{~m}$ deep. It is equipped with a multidirectional wavemaker with 32 triangular plungers (0.31 $\mathrm{m}$ wide) [31]. A sloping beach is deployed opposite the wavemaker to absorb the wave energy. The tank is also equipped with a pump (located beside the basin) for the generation of background currents up to $0.4 \mathrm{~m} / \mathrm{s}$; the stream can follow or oppose the waves. One of the inlet/outlet is located below the beach, while a second is located below the wavemaker (approximately $2 \mathrm{~m}$ below the water level). Note that no modification of the cross section was performed to locally modify the velocity field. Wave probes were deployed along the tank at a distance of $2.5 \mathrm{~m}$ from the sidewall and arranged at $5 \mathrm{~m}$ intervals to monitor the evolution of wave trains (a six-probe array was also deployed to monitor directional properties); for consistency with the wave flume experiments, only probes within $25 \mathrm{~m}$ from the wavemaker were considered, though. Two electromagnetic velocimeters were used to survey the current. Instruments were deployed at several locations in the tank at a depth of $0.2 \mathrm{~m}$; a vertical profile was measured at about $10 \mathrm{~m}$ from the wavemaker. Instantaneous measurements of horizontal velocity revealed that the flow had substantial spatial and temporal variations, with a dominant oscillation period of approximately $150 \mathrm{~s}$. Average values are presented in Figs. 11 and 1 d. For these tests, the standard deviation was about $25 \%$ of the mean over the entire time series. As the flow's outlet is located just below the wave generator, the velocity is approximately zero at a distance of about $0.2 \mathrm{~m}$ from the wavemaker, while the flow is at regime at a distance of $5 \mathrm{~m}$ from the wavemaker. Waves are therefore generated in a condition of (almost) still water and undergo a current gradient about $1 \mathrm{~m}$ after being generated. Farther from the wavemaker, between 5 and $30 \mathrm{~m}$ from the generator, the current still shows a weak gradient, which may slightly affect the wave field. Although the average horizontal velocity weakly decreased with the water depth, the vertical profile remained fairly uniform over a depth of about $1.5 \mathrm{~m}$. The survey of the current field also indicated that the current runs faster on one side (along the wave probes), while it is slower on the other (see Fig. 1, for example). It is important to mention that the crosstank gradient refracts the wave field towards the sidewall (probes side). As such, this may cause linear directional focusing, steepening the wave profile and hence affecting wave dynamics.

A number of tests characterized by different values of the modulation frequency and current velocity have been carried out in both facilities. For all tests, the initial signal at the wavemaker consisted of a three-component 

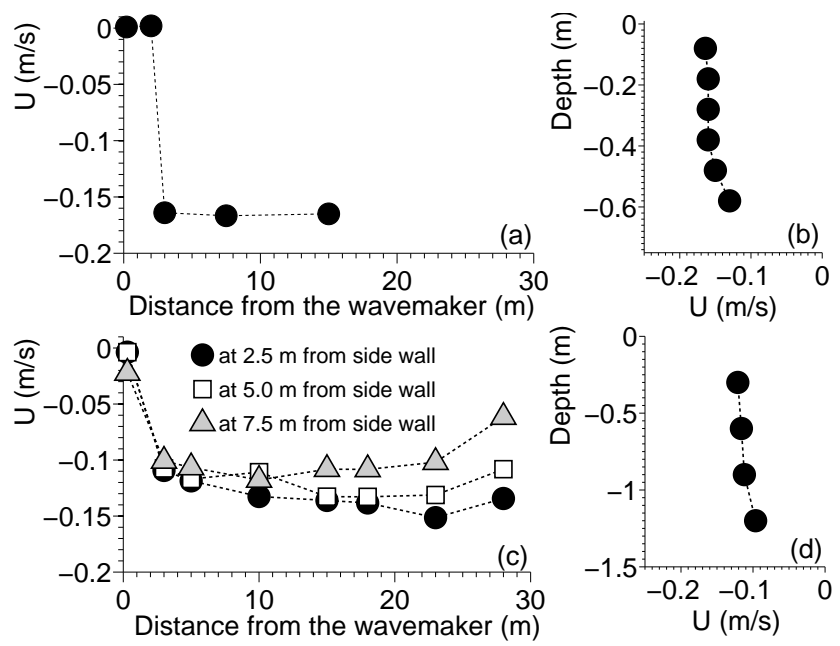

FIG. 1: Average horizontal velocity for a current field opposing wave propagation: longitudinal (a) and vertical (b) profiles in the wave flume of Plymouth University; and longitudinal (c) and vertical (d) profiles in the directional wave basin of the University of Tokyo. The longitudinal profiles were measured at a depth of $0.3 \mathrm{~m}$ at the University of Tokyo and $0.08 m$ at Plymouth University.

system: a carrier wave of period $T_{0}=0.8 \mathrm{~s}$ (wavelength $\left.\lambda_{0}=2 \pi / k_{0} \simeq 1 \mathrm{~m}\right)$ and two side bands with amplitudes $b_{ \pm}$equal to 0.25 times the amplitude $a_{c}$ of the carrier waves. Considering the water depth of $0.75 \mathrm{~m}$ in the wave flume and $5 \mathrm{~m}$ in the directional basin, experiments were performed under deep water conditions $\left(k_{0} h>4\right)$. As the effect of the current is to steepen the wave profile, a small initial steepness was selected in order to avoid wave breaking. The tests presented here were carried out by selecting the wave amplitude of the carrier wave $a_{c}$ in such a way that the wave steepness was $k_{0} a_{0}=0.063$ with $a_{0}^{2}=a_{c}^{2}+b_{+}^{2}+b_{-}^{2}$. The frequency of the disturbances was chosen to force the number of waves under the perturbation $N=\omega_{0} / \Delta \Omega$ (with $\omega_{0}$ being the angular frequency of the carrier waves) to be equal to 11 . Under these circumstances, the perturbation frequency lays just outside the NLS-based instability region, i.e. waves are stable $\left(\varepsilon N=k_{0} a_{0} N=0.69<1 / \sqrt{2}\right)$. These packets were tested against different opposing current speeds. Velocities ranged from $0 \mathrm{~m} / \mathrm{s}$ to $-0.30 \mathrm{~m} / \mathrm{s}$ with step of $-0.02 \mathrm{~m} / \mathrm{s}$. All tests were run for a time period of 10 minutes. Considering the variability of the current in the directional basin, this ensured enough data to perform a standard statistical analysis of the observations.

Typical time series of the water surface elevation are presented in Fig. 2 for $U_{0} / c_{g}=0$ and $U_{0} / c_{g}=-0.1$. What is clear from the figure is that, while the modulation does not grow in absence of current (top panels), the wave packets undergo a modulationally unstable process (nonlinear focussing) and wave amplification in the presence of a current. Interestingly enough, amplification is less sharp in the wave flume due to a more regular current field and the absence of contaminating three-dimensional
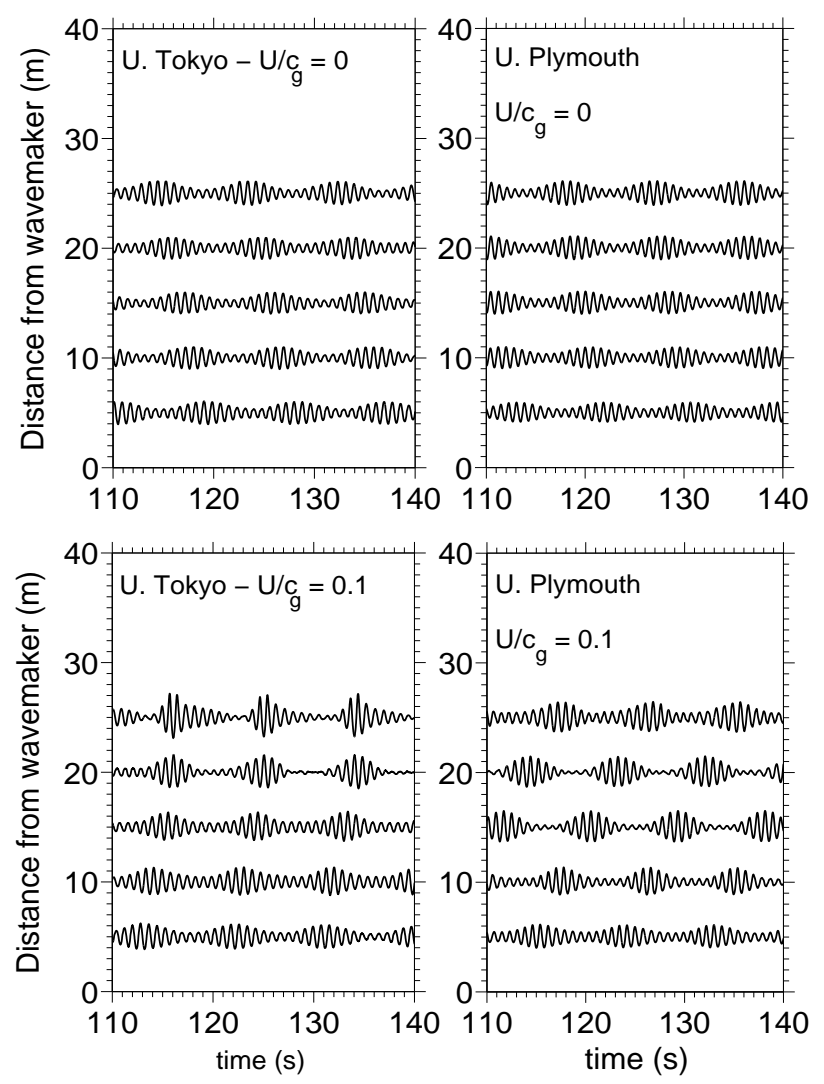

FIG. 2: Evolution of surface elevation: samples from the wave flume of Plymouth University (right panels) and the directional wave basin of the University of Tokyo (left panels).

effects.

A standard zero-crossing procedure was applied to extract the maximum amplitude at each probe. Because of the temporal variability of the current in the directional basin, the analysis was performed on segments of three consecutive wave groups, i.e. time window $\tau=26.4 \mathrm{~s}$, where the current was nearly steady (velocity fluctuations were confined within a range of $\pm 0.01 \mathrm{~m} / \mathrm{s}$, namely one order of magnitude smaller than the average). As the prediction in equation (2) only includes the contribution of free wave modes, frequencies greater than $1.5 \omega_{c}$ and smaller than $0.5 \omega_{c}$ were removed to filter out bound modes. The amplitude was then normalised by $E^{1 / 2}=\left[(1 / \tau) \int_{0}^{\tau}|A|^{2} d t\right]^{1 / 2}$, where $A$ is the wave envelope of the concurrent segment, to eliminate the current-induced increase of wave amplitude. An average normalised maximum amplitude and standard deviation were calculated over the entire time series. The maximum wave amplitude is presented as a function of $U_{0} / c_{g}$ in Figure 3 together with equation (2) (solid line) and the prediction model in (3) (dashed line); error bands equivalent to the $68 \%$ confidence interval (one standard deviation) are also shown; owing to the stable current field, the error band for flume experiments is notably smaller than the one detected in the directional basin. 


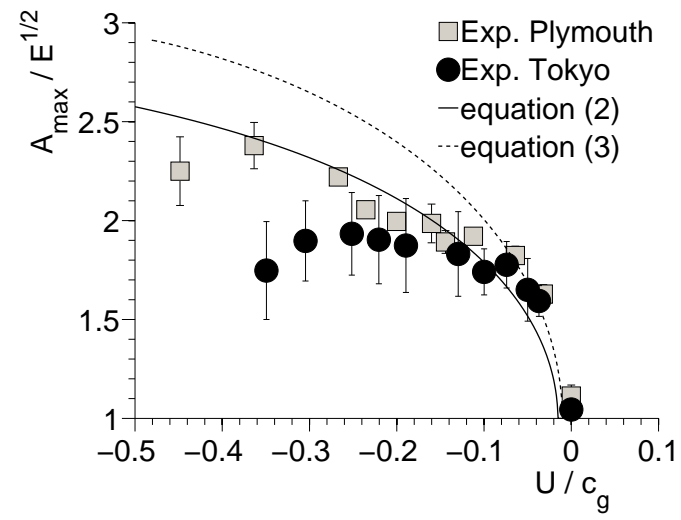

FIG. 3: Normalised maximum amplitude as a function of $U_{0} / c_{g}$ : equation (2), solid line; equation (2) modified by including the conservation of wave action, dashed line.

Qualitatively, both tests are in good agreement with theory, substantiating that an adverse current gradient triggers moduational instability processes. Quantitatively, however, observations are notably overestimated by the model in (3), especially for strong current fields. Equation (2), on the contrary, produces a satisfactory approximation of the records, particularly for the flume experiments. It is important to remark, in this regard, that three dimensional effects induced by refraction enhance the breaking probability in the directional wave basin and hence limit the maximum wave amplitude also for relatively mild currents. Amplitude growth ceases, in fact, for $\left|U_{0} / c_{g}\right|>0.2$ as individual waves systematically reach their limiting steepness [32] and break. In the wave flume, three-dimensional effects are suppressed, making breaking dissipation less likely and thus allowing waves to develop the maximum amplification. This justifies the good agreement with theory until $\left|U_{0} / c_{g}\right| \approx 0.4$; beyond this threshold waves reach the limiting steepness and break.

In conclusion, the conjecture on the generation of rogue waves in opposite current using a current-modified cubic NLS equation has been here confirmed by two independent sets of laboratory experiments, which were carried out in a wave flume and a narrow directional wave basin. Observations corroborate that the excitation of the modulation and the concurrent intensification of the maximum wave growth is a function of the adverse current $U_{0} / c_{g}$ and is consistent with theoretical predictions in equation (2), but overestimated by the model in (3). Despite possible shortcomings of the theory and uncer- tainties in the experimental conditions, especially related to the irregularities of the current field, the present study clearly shows evidence that an opposing current field can destabilize an otherwise stable wave packet. The result is the formation of a rogue wave, whose amplitude depends on the ratio of current velocity to group velocity. Note that the essence of the theoretical predictions in [28] and [30] is to account for the impact of the current shear only to modify the basic parameters that control the dynamics of the wave train. Once the basic parameters have changed, the instability process follows its nominal evolution pattern (namely, the nonlinear stages of modulational instability). It is important to mention that this is the case for our experiments because the spatial scale of the current field variation was smaller than the spatial scale of the nonlinear evolution. This is not necessarily the case in reality and further research is needed for those cases when the two scales are of the same order. From a physical point of view, the process that we have described may take place in nature when a modulationally stable swell (waves propagating without the forcing of the wind), which is characterized by a narrow spectrum (both in direction and frequency) enters a region of an opposite variable current. Besides the linear effect of refraction which could generate linear focussing, the current gradient can destabilise the wave packets leading to a nonlinear focussing effect and the formation of rogue waves. Indeed, as mentioned in [28], such currents may reach velocities up to $1.5 \mathrm{~m} / \mathrm{s}$, and for a group velocity corresponding to waves of period equal to $10 \mathrm{~s}$ (a typical condition during storms), the ratio $U_{0} / c_{g}$ is of the order of 0.2 , large enough to trigger a dangerous rogue wave. We believe that the mechanism we have observed is universal and can be reproduced in all fields of physics where an NLS equation with variable coefficient of the nonlinear term can be written.

Acknowledgments Experiments were supported by the JSPS Fellowship for Research in Japan Program, Grants-in-Aid for Scientific Research of the JSPS and the International Science Linkages (ISL) Program of the Australian Academy of Science. Financial support of the Australian Research Council and Woodside Energy Ltd through the grant LP0883888 is also acknowledged. M. O. and A. T. acknowledge the VDRS grant from Swinburne University of Technology. M.O. was supported by EU project EXTREME SEAS (SCP8-GA-2009-234175) and ONR grant N000141010991. M.O. acknowledges Dr. B. GiuliNico for interesting discussions.
[1] V. Zakharov and A. Dyachenko, European Journal of Mechanics-B/Fluids 29, 127 (2010).

[2] A. Newell and J. Moloney, Nonlinear optics (AddisonWesley Redwood City, California, 1992).

[3] V. Zakharov, S. Musher, and A. Rubenchik, Physics re- ports 129, 285 (1985).

[4] V. Zakharov and A. Shabat, Functional Analysis and Its Applications 8, 226 (1974).

[5] E. Kuznetsov, in Akademiia Nauk SSSR Doklady (1977), vol. 236, pp. 575-577, ISSN 0002-3264. 
[6] N. Akhmediev, V. Eleonskii, and N. Kulagin, Theor. Math. Phys. 72, 809 (1987), ISSN 0040-5779.

[7] D. Peregrine, The Journal of the Australian Mathematical Society. Series B. Applied Mathematics 25, 16 (1983).

[8] A. Chabchoub, N. Hoffmann, and N. Akhmediev, Physical Review Letters 106, 204502 (2011).

[9] A. Chabchoub, N. Hoffmann, M. Onorato, and N. Akhmediev, Physical Review X 2, 011015 (2012).

[10] B. Kibler, J. Fatome, C. Finot, G. Millot, G. Genty, B. Wetzel, N. Akhmediev, F. Dias, and J. Dudley, Scientific reports 2 (2012).

[11] B. Kibler, J. Fatome, C. Finot, G. Millot, F. Dias, G. Genty, N. Akhmediev, and J. Dudley, Nature Physics 6, 790 (2010).

[12] H. Bailung, S. K. Sharma, and Y. Nakamura, Phys. Rev. Lett. 107, 255005 (2011), URL http://link.aps.org/doi/10.1103/PhysRevLett.107.255005

[13] K. B. Dysthe and K. Trulsen, Physica Scripta T82, 48 (1999).

[14] A. Osborne, M. Onorato, and M. Serio, Phys. Lett. A 275, 386 (2000).

[15] V. Zakharov and L. Ostrovsky, Physica D: Nonlinear Phenomena 238, 540 (2009).

[16] Z. Yan, Physics Letters A 374, 672 (2010).

[17] C. Dai, X. Wang, and J. Zhang, Annals of Physics 326, 645 (2011).

[18] M. Onorato and D. Proment, Physics Letters A 376, 3057 (2012), ISSN 0375-9601, URL http://www.sciencedirect.com/science/article/pii/S0375960112008377.
[19] A. Chawla, Ph.d. thesis, University of Delaware (USA) (2000).

[20] A. Chawla and J. T. Kirby, J. Geophys. Res. 107 (2002).

[21] M. Gerber, J. Fluid Mech. 176, 311 (1987).

[22] R. J. Lai, S. R. Long, and N. Huang, J. Geophysic. Res. 94, 16201 (1989).

[23] R. Moreira and D. Peregrine, J. Fluid Mech. 691, 1 (2012).

[24] J. D. Stocker and D. H. Peregrine, J. Fluid Mech. 399, 335 (1999).

[25] I. K. Suastika, Ph.d. thesis, Technische Universiteit Delft (The Netherlands) (2004).

[26] A. Toffoli, L. Cavaleri, A. V. Babanin, M. Benoit, E. M. Bitner-Gregersen, J. Monbaliu, M. Onorato, A. R. Osborne, and C. T. Stansberg, Nat. Hazards Earth Syst. Sci. 11, 1 (2011).

K. B. Hjelmervik and K. Trulsen, J. Fluid Mech. 637, 267 (2009).

[28] M. Onorato, D. Proment, and A. Toffoli, Phys. Rev. Lett. 107, 184502 (2011), URL http://link.aps.org/doi/10.1103/PhysRevLett.107.184502,

[29] L. Ying, Z. Zhuang, E. Heller, and L. Kaplan, Nonlinearity 24, R67 (2011).

[30] V. Ruban, JETP letters 95, 486 (2012).

[31] T. Waseda, T. Kinoshita, and H. Tamura, J. Phys. Oceanogr. 39, 621 (2009).

[32] A. Toffoli, A. V. Babanin, M. Onorato, and T. Waseda, Geophys. Res. Lett. 37, L05603 (2010). 